\title{
Warburg Therapy
}

National Cancer Institute

\section{Source}

National Cancer Institute. Warburg Therapy. NCI Thesaurus. Code C29545.

The cornerstone of oxygenation therapy is the presumption that human disease, including cancer, is caused by a deficit of tissue oxygen. According to proponents, hypoxia results in anaerobic fermentation, a loss of capacity for oxidative detoxification of toxins and metabolic products, and failure of immune killing of invading bacteria and viruses. To restore ability to carry out these functions, oxyg enation promoters propose using chemicals they claim will release oxygen in tissue or act as germicides in vivo. 